\title{
Blaschkoid hyperkeratotic plaques in a hemi-distribution since infancy
}

Logan W. Thomas, MS4, ${ }^{a}$ Ashley N. Elsensohn, MD, MPH, ${ }^{a}$ Jessica Shiu, MD, PhD, ${ }^{a}$ Sebastien de Feraudy, MD, PhD, ${ }^{a}$ and Frances Segal, $\mathrm{MD}^{\mathrm{b}}$ Irvine and Mission Viejo, California
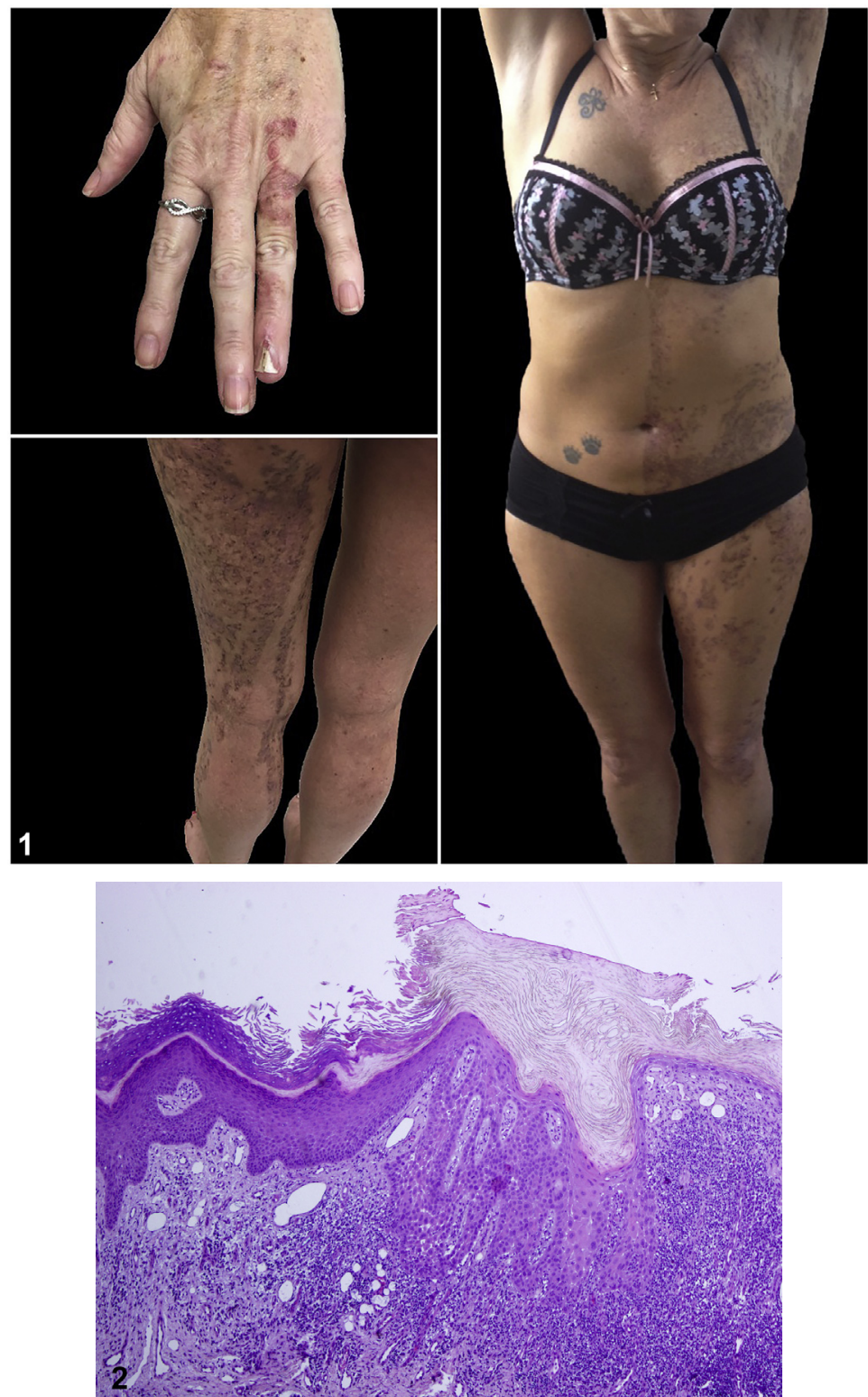

From the Department of Dermatology, University of California, Irvine ${ }^{\mathrm{a}}$ and Integrated Dermatology of Mission Viejo. ${ }^{\mathrm{b}}$

Funding sources: None.

Conflicts of interest: None declared.

Correspondence to: Ashley N. Elsensohn, MD, MPH, Department of Dermatology, University of California Irvine, 18 Medical Surge I, Irvine, CA 92697-2400. E-mail: aelsenso@uci.edu.
JAAD Case Reports 2018;4:402-4.

2352-5126

(c) 2017 by the American Academy of Dermatology, Inc. Published by Elsevier, Inc. This is an open access article under the CC BY-NC-ND license (http://creativecommons.org/licenses/by-nc$\mathrm{nd} / 4.0 /$ ).

http://dx.doi.org/10.1016/j.jdcr.2017.08.013 
A 41-year-old woman presented with brown hyperkeratotic plaques in a blaschkoid distribution on the left side of her body, including face, neck, torso, arm, hand, leg, and foot (Fig 1). There was a sharp line of delineation in the midabdomen between normal and pathologic skin. The patient reported having these lesions since the age of 1 year. She noted difficulty healing and also noted that upon injury to the lesion, wounds would heal thicker, rougher, and darker. She denied family history of skin disease. A biopsy specimen of the left arm was obtained and stained with hematoxylin-eosin (Fig 2).

\section{Question 1: What is your diagnosis?}

A. Lichen planus (LP)

B. Incontinentia pigmenti

C. Linear epidermal nevus

D. Linear porokeratosis with dysplasia

E. Darier disease

\section{Answers:}

A. LP - Incorrect. LP typically has deeper purple, polygonal papules with fine white lines and characteristic Wickham striae. LP most commonly occurs on the limbs and dorsal aspects of the trunk, with nails and mucous membranes commonly involved. ${ }^{1}$

B. Incontinentia pigmenti - Incorrect. Incontinentia pigmenti usually occurs in female infants with a staged presentation progressing in order from vesicular, verrucous, hyperpigmented, and hypopigmented and atrophic blaschkoid lesions. Abnormalities can also be seen in teeth, hair, and nails. One-third of patients have ocular and neurologic abnormalities. ${ }^{2}$

C. Linear epidermal nevus - Incorrect. Linear epidermal nevi are benign hamartomatous growths of epidermal cells that typically occur at birth. Lesions are described as brown- to skin-colored plaques, and patches can be Blaschkoid and often have a midline distribution. ${ }^{3}$

D. Linear porokeratosis with dysplasia - Correct. Porokeratosis results from abnormal keratinization and presents in numerous phenotypes, such as disseminated superficial actinic porokeratosis of Mibelli, or punctate. Linear porokeratosis accounts for $3.5 \%$ to $15 \%$ of all cases of pororkeratosis. With a slight female predominance, linear porokeratosis normally presents at early childhood as an eruption following the lines of Blaschko. ${ }^{4}$

E. Darier disease - Incorrect. Darier disease presents as skin-colored papules in a seborrheic distribution normally on the face and chest with lesions typically having a rough texture resembling sandpaper. ${ }^{5}$

\section{Question 2: What is the pathognomonic histo- logic finding of porokeratosis?}
A. Jagged saw tooth acanthosis
B. Cornoid lamella
C. Abundant melanophages
D. Papillomatosis and hyperkeratosis
E. Corp grains

\section{Answers:}

A. Jagged saw tooth acanthosis - Incorrect. Jagged sawtooth acanthosis is a feature of LP. ${ }^{1}$

B. Cornoid lamella - Correct. Classic histology for porokeratosis shows a coronoid lamella at a $45^{\circ}$ angle, hyperkeratosis, and either atrophy or acanthosis of the central lesion. ${ }^{4}$ However, in longstanding porokeratosis, the classic cornoid lamella can be difficult to identify. This patient's histology showed moderate-to-severe cytologic atypia of keratinocytes, present through most of the epidermis. The illustrative biopsy is that of squamous cell carcinoma (SCC) in situ on a background of long-standing linear porokeratosis (Fig 2). Of note, this patient had numerous SCCs that were treated with topical 5-fluorouracil and electrocurettage and desiccation. Probability of malignant transformation ranges from $7.5 \%$ to $19 \%$ in long-term follow up-the larger and longer the lesion, the greater the risk of malignant transformation. ${ }^{4}$

C. Abundant melanophages - Incorrect. Abundant melanophages are seen in incontinentia pigmenti. ${ }^{2}$

D. Papillomatosis and hyperkeratosis - Incorrect. Epidermal nevi are characterized by hyperkeratosis, acanthosis, and papillomatosis. ${ }^{3}$ 
E. Corp grains - Incorrect. Dariers disease is characterized by corp grains and ronds, dyskeratotic cells with elongated pyknotic nuclei, and acantholytic cells in lacunae, respectively. ${ }^{5}$

Question 3: The patient noted difficulty healing and that upon injury to the lesion, wounds would heal thicker, rougher, and darker. What is the significance of this statement?
A. Postinflammatory hyperpigmentation
B. Koebner phenomenon
C. Keloid formation
D. Scar formation
E. SCC

\section{Answers:}

A. Postinflammatory hyperpigmentation - Incorrect. Common reaction of body to inflammation or injury however does not commonly result in thicker and rougher lesions.

B. Koebner phenomenon - Correct. The Koebner phenomenon is defined as spreading of the lesion to uninvolved skin through trauma, dermatoses, or therapeutics. The patient most likely was experiencing spread of her linear porokeratosis after trauma to unaffected skin. The Koebner phenomenon has been described in many dermatologic disease, one of which includes porokeratosis of Mibelli and disseminated superficial actinic porokeratosis. ${ }^{6}$ No reported cases of Koebnerization have been reported with linear porokeratosis, but this is likely a phenomenon as with other subtypes of porokeratosis.

C. Keloid formation - Incorrect. Keloids are overgrowth of scar tissue that exceeds the scar margins. Keloids can heal thicker and darker but are typically smooth and firm in nature. Our patient did not have any history of keloid formation.

D. Scar formation - Incorrect. Scar formation is a definite possibility, but usually scars are not initially pigmented or rough.

E. SCC - Incorrect. SCCs are more likely to result in those patients with linear porokeratosis; however, the description does not coincide with a typical description of SCC. SCCs have multiple presentations but usually present as a hyperkeratotic, erythematous papule or nodule that may ulcerate.

\section{Abbreviations used:}

LP: lichen planus

SCC: squamous cell carcinoma

\section{REFERENCES}

1. Weston G, Payette M. Update on lichen planus and its clinical variants. Int J Womens Dermatol. 2015;1(3):140-149.

2. Landy SJ, Donnai D. Incontinentia pigmenti (Bloch-Sulzberger syndrome). J Med Genet. 1993;30:53-59.

3. Brandling-Bennett HA, Morel KD. Epidermal nevi. Pediatr Clin North Am. 2010;57(5):1177-1198.

4. Martorell-Calatayud A, Blanes M, Requena C, Botella-Estrada R, Guillén-Barona C. Generalized linear porokeratosis limited to one side of the body. Actas Dermosifiliogr. 2009;100(10):911-913.

5. Takagi A, Kamijo M, Ikeda S. Darier disease. J Dermatol. 2016;43:275-279.

6. Sagi L, Trau H. The Koebner phenomenon. Clin Dermatol. 2011;29(2):231-236. 